BULLETIN (New Series) OF THE

AMERICAN MATHEMATICAL SOCIETY

Volume 38, Number 4, Pages 409-433

S 0273-0979(01)00917-X

Article electronically published on June 12, 2001

\title{
WHICH ARE THE SIMPLEST ALGEBRAIC VARIETIES?
}

\author{
JÁNOS KOLLÁR
}

\begin{abstract}
This paper is a slightly revised version of the notes prepared in connection with the AMS Colloquium Lectures delivered in New Orleans, January 2001.
\end{abstract}

\section{Contents}

1. Introduction 409

2. Algebraic curves $\quad 410$

3. Rational surfaces $\quad 417$

4. Minimal models 423

5. Rationally connected varieties $\quad 424$

6. Rationally connected varieties over $\mathbb{R} \quad 426$

7. Open problems $\quad 430$

References

\section{INTRODUCTION}

An algebraic variety is a subset of $\mathbb{C}^{n}$ defined by polynomial equations. It is rather clear that the higher the degree of the defining equations, the more complicated the corresponding variety can be. There have been various approaches to define what the "most complicated" varieties are, but it is only recently that a good definition and theory were developed for the "simplest" varieties. These are called rationally connected varieties.

The first 4 sections are devoted to motivating the general definition with classical examples of curves and surfaces.

Rationally connected varieties are finally defined in section 5. A list of their known good properties is also given. In dimension 3, rationally connected varieties are known to have all the expected properties. In higher dimensions some of the hoped for results are still open, but no counter examples are known.

Section 6 studies a conjecture of Nash about the real topology of rationally connected varieties. Finally, several open problems are stated in section 7 .

Received by the editors February 7, 2001.

2000 Mathematics Subject Classification. Primary 14-01, 14E08, 14E30, 14G05, 14J26, 14P25; Secondary 11D25, 11G35, 30F10, 57N10. 


\section{Algebraic Curves}

The study of polynomial equations in 2 variables

$$
f(x, y)=0
$$

has a long and distinguished history. As motivation for the higher dimensional theory, I would like to concentrate on a few aspects of it that have attracted a lot of attention.

1 (Number theory). Several well known problems of number theory are equivalent to a question about rational solutions of an equation $f(x, y)=0$. For instance, solving the Fermat equation

$$
x^{n}+y^{n}=z^{n}
$$

in integers is equivalent to solving

$$
x^{n}+y^{n}-1=0
$$

in rationals.

2 (Complex analysis and topology). If we let $x$ and $y$ be complex variables, the solutions of $f(x, y)=0$ in $\mathbb{C}^{2}$ give a Riemann surface. This is the beginning of complex manifold theory and one of the starting points of topology.

3 (Real topology). The real solutions of $f(x, y)=0$ give a real curve in $\mathbb{R}^{2}$. Understanding the complexity of these curves is Hilbert's 16th problem.

4 (Integration). Integrals of algebraic functions have been a major subject of study in the XIXth century. For instance, integrals of the form

$$
\int \frac{h(x)}{\sqrt[n]{g(x)}} d x
$$

lead to the study of the curve $y^{n}-g(x)=0$. From the modern perspective, these results are the beginnings of global differential geometry of complex manifolds.

As an illustration, I would like to analyze in some detail the equations

$$
y^{2}=f_{m}(x) \text { where } f_{m} \text { is a polynomial of degree } m \text {. }
$$

These are called hyperelliptic curves or equations. We assume that $f_{m}$ has no multiple roots. For most questions this can be achieved easily. Indeed, if $f(x)=$ $(x-a)^{2} g(x)$, then a substitution $y_{1}=y /(x-a)$ gives the simpler equation

$$
y_{1}^{2}=g(x) .
$$

The main point that I would like to stress is the following.

Claim 5. The equations $y^{2}=f_{m}(x)$ naturally break up into 3 groups of quite different flavor:

1. (Simple cases) $m=1,2$

2. (Intermediate cases) $m=3,4$

3. (Hard cases) $m \geq 5$.

A key element of this claim is that all 4 of the above viewpoints result in the same division into groups. Let us begin with the most transparent aspect. 
6 (Topology of the complex solutions). Here we start with the set

$$
H_{m}:=\left\{(x, y) \in \mathbb{C}^{2}: y^{2}=f_{m}(x)\right\} .
$$

For large values of $x, H_{m}$ is like $\left(y^{2}=x^{m}\right)$. If $m$ is odd, then the latter is a connected set, parametrized by the unit disc via $t \mapsto\left(t^{-m}, t^{-2}\right)$. Thus

$$
\bar{H}_{m}:=H_{m} \cup\{\text { one point at infinity }\}
$$

is a compact topological surface.

If $m$ is even, then $\left(y^{2}=x^{m}\right)$ is disconnected near infinity, parametrized by 2 copies of the unit disc via $t \mapsto\left( \pm t^{-m / 2}, t^{-1}\right)$. Thus

$$
\bar{H}_{m}:=H_{m} \cup\{\text { two points at infinity }\}
$$

is a compact topological surface.

The projection to the $x$-axis establishes $\bar{H}_{m}$ as a 2-sheeted ramified cover of the Riemann sphere $S^{2}=\mathbb{C} \cup\{\infty\}$. The points in the Riemann sphere with 1 preimage are the roots of $f_{m}$ and the point at infinity for $m$ odd.

Choose a triangulation of the Riemann sphere where the roots of $f_{m}$ and the point at infinity are vertices. The Euler characteristic formula gives that

$$
2=\chi\left(S^{2}\right)=v-e+r,
$$

where $v$ is the number of vertices, $e$ is the number of edges and $r$ the number of regions in the triangulation. Pulling this back to $\bar{H}_{m}$, we obtain a triangulation with $r^{\prime}=2 r$ regions, $e^{\prime}=2 e$ edges and $v^{\prime}=2 v-m-(0$ or 1$)$ vertices (depending on the parity of $m$ ). Thus

$$
\chi\left(\bar{H}_{m}\right)= \begin{cases}1-m & \text { if } m \text { is odd } \\ 2-m & \text { if } m \text { is even. }\end{cases}
$$

It is easy to see that $\bar{H}_{m}$ is orientable. From the classification of compact topological surfaces we obtain that $\bar{H}_{m}$ is $S^{2}$ with $\left\lfloor\frac{m-1}{2}\right\rfloor$ handles attached. The number of handles is called the genus; it is usually denoted by $g=g\left(\bar{H}_{m}\right)$. Thus, topologically,

$$
\bar{H}_{m} \sim\left\{\begin{array}{l}
\text { sphere if } m=1,2 \\
\text { torus if } m=3,4 \\
\text { surface of genus } \geq 2 \text { if } m \geq 5 .
\end{array}\right.
$$

Relying on more complex analysis, we can conclude that the universal cover of $\bar{H}_{m}$ is biholomorphic to

$$
\left\{\begin{array}{l}
\text { Riemann sphere if } m=1,2 \\
\text { complex plane if } m=3,4 \\
\text { complex unit disc if } m \geq 5 .
\end{array}\right.
$$

Next we look at the real topology. This is easy but the division into groups is less convincing.

7 (Real topology). Let $a_{1} \leq \cdots \leq a_{n}$ be the real roots of $f_{m}$. If $f_{m}$ is positive in the interval $\left(a_{i}, a_{i+1}\right)$, then the graph of $y^{2}=f_{m}$ over this interval is a circle. It is also easy to see what happens near infinity. The end result is that the real points of $\bar{H}_{m}$ form at most $\left\lfloor\frac{m+1}{2}\right\rfloor$ circles. Thus here we cannot determine $m$ from the topology of the real solutions, but we have an inequality

$$
\text { \#(connected components of } \left.\bar{H}_{m}(\mathbb{R})\right) \leq g+1=\left\lfloor\frac{m+1}{2}\right\rfloor .
$$


8 (Rational solutions). If $m=1$, then $y^{2}=a x+b$ can be solved for $x$ and we always have plenty of rational solutions parametrized as

$$
t \mapsto\left(\frac{t^{2}-b}{a}, t\right) .
$$

If $m=2$, then $y^{2}=a x^{2}+b x+c$ may not have any rational solutions. (For instance, $y^{2}=-x^{2}-1$ has no solutions even over $\mathbb{R} ; y^{2}=-x^{2}+3$ has real solutions but no rational solutions, as a simple mod 3 argument shows.) I claim, however, that as soon as we have one rational solution, there are plenty more. Look for instance at $y^{2}+2 x^{2}=3$. One obvious solution is $(1,1)$. Let $L_{t}$ be the line connecting $(1,1)$ with $(1+t, 0) . L_{t}$ intersects the ellipse $\left(y^{2}+2 x^{2}=3\right)$ in 2 points; one of these is $(1,1)$. The coordinates of the other point give rational solutions parametrized as

$$
t \mapsto p(t)=\left(\frac{-2 t^{2}+2 t+1}{2 t^{2}+1}, \frac{2 t^{2}+4 t-1}{2 t^{2}+1}\right) .
$$

We get Figure 1

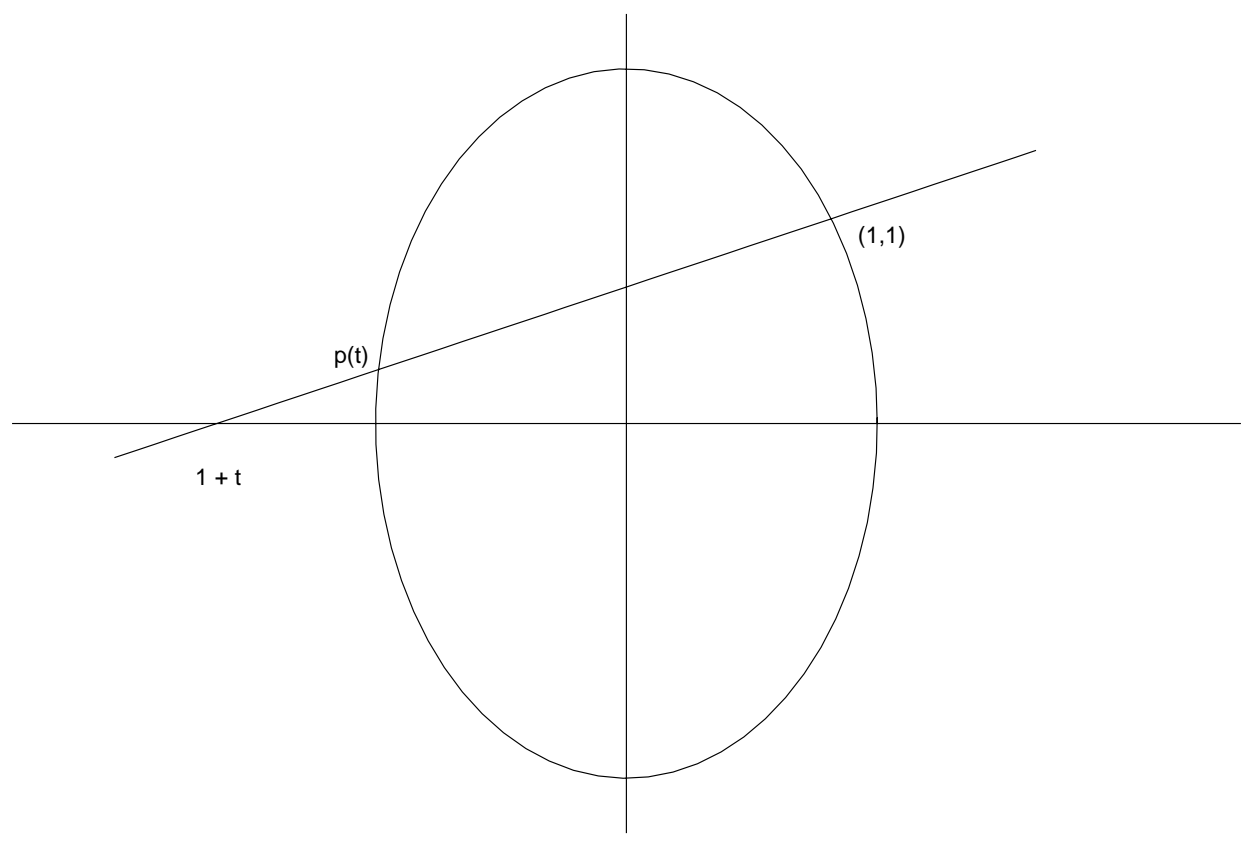

Figure 1. Parametrization of $y^{2}+2 x^{2}=3$

We may summarize these results as follows:

Stereographic projection of a conic $q(x, y)=0$ from a point $P$ on the conic gives a one-to-one correspondence between the points on the conic and the points of a line.

The inverse is given by quotients of polynomials of degree 2. The coefficients of these polynomials are in the same field as the coefficients of $q(x, y)$ and the coordinates of $P$. 


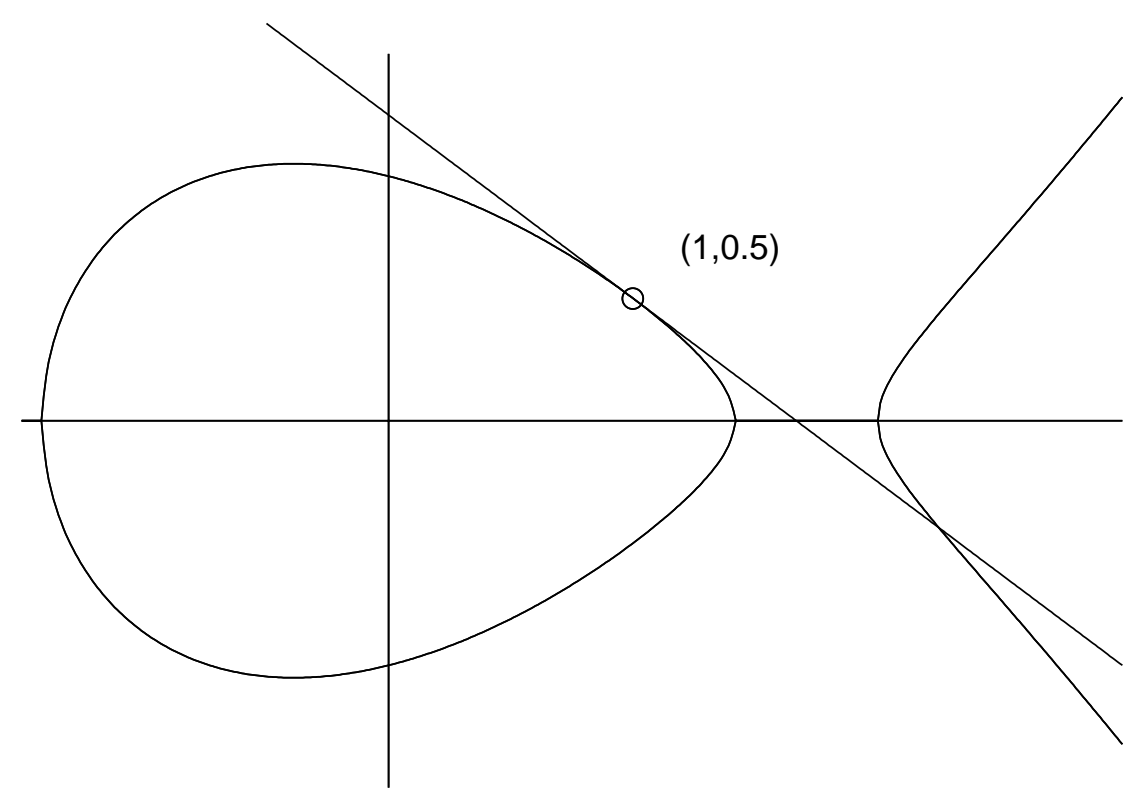

Figure 2. Tangent method for $4 y^{2}=x^{3}-2 x^{2}-2 x+4$

If $m=3$, then there is an interesting method to get further solutions from any given one. This relies on the observation that

$$
H_{3}=\left(y^{2}=x^{3}+a x^{2}+b x+c\right) \subset \mathbb{C}^{2}
$$

is a degree 3 curve, so any line intersects it in at most 3 points. If $\left(x^{\prime}, y^{\prime}\right)$ and $\left(x^{\prime \prime}, y^{\prime \prime}\right)$ are points on $H_{3}$, the line connecting them intersects $H_{3}$ in a unique third point, $\left(\phi_{x}\left(x^{\prime}, x^{\prime \prime}\right), \phi_{y}\left(y^{\prime}, y^{\prime \prime}\right)\right)$. Moreover, if $a, b, c, d, x^{\prime}, y^{\prime}, x^{\prime \prime}, y^{\prime \prime}$ are rational numbers, then so are $\phi_{x}\left(x^{\prime}, x^{\prime \prime}\right)$ and $\phi_{y}\left(y^{\prime}, y^{\prime \prime}\right)$. We can even allow $\left(x^{\prime \prime}, y^{\prime \prime}\right)=\left(x^{\prime}, y^{\prime}\right)$ by using the tangent line at $\left(x^{\prime}, y^{\prime}\right)$ to $H_{3}$. See Figure 2.

Thus, starting with a point $\left(x_{0}, y_{0}\right) \in H_{3}$ we obtain

$$
\left(x_{1}, y_{1}\right)=\left(\phi_{x}\left(x_{0}, x_{0}\right), \phi_{y}\left(y_{0}, y_{0}\right)\right) \in H_{3} .
$$

By explicit computation we obtain that

$$
\begin{aligned}
& x_{1}=x_{0}-\frac{1}{4} \frac{4 x_{0}^{3} a+6 x_{0}^{2} b+12 x_{0} c+4 a c+3 x_{0}^{4}-b^{2}}{y_{0}^{2}} \\
& y_{1}=y_{0}-\frac{1}{8} \frac{\left(4 x_{0}^{3} a+6 x_{0}^{2} b+12 x_{0} c+4 a c+3 x_{0}^{4}-b^{2}\right)\left(3 x_{0}^{2}+2 a x_{0}+b\right)}{y_{0}^{3}} .
\end{aligned}
$$

This procedure can be iterated to obtain points

$$
\left(x_{i+1}, y_{i+1}\right)=\left(\phi_{x}\left(x_{0}, x_{i}\right), \phi_{y}\left(y_{0}, y_{i}\right)\right) \in H_{m} .
$$

In some cases we get back to $\left(x_{0}, y_{0}\right)$ and then we just go around in circles, but in most cases we do get an infinite sequence of points. By looking carefully at the above formulas we find that

The numerators and denominators of $\left(x_{i}, y_{i}\right)$ grow exponentially with $i$.

If $m=4$, then a line intersects

$$
H_{4}=\left(y^{2}=x^{4}+a x^{3}+b x^{2}+c x+d\right)
$$




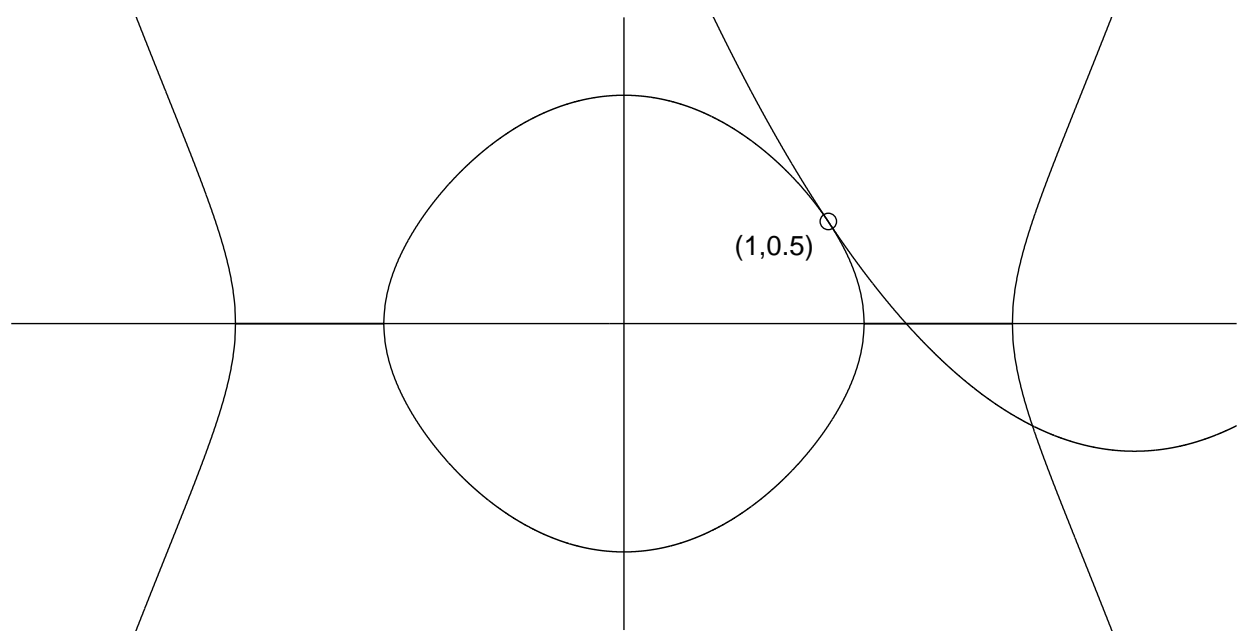

Figure 3. Tangent method for $4 y^{2}=x^{4}-5 x^{2}+5$

in 4 points; thus the above tangent method does not work. One can, however, modify it as follows. It is easy to see that every parabola of the form $y=x^{2}+A x+B$ intersects $H_{4}$ in at most 3 points, and for every $\left(x_{0}, y_{0}\right) \in H_{4}$ there is a unique parabola $y=x^{2}+A x+B$ which is tangent to $H_{4}$ at $\left(x_{0}, y_{0}\right)$. The 3 rd intersection point is then $\left(x_{1}, y_{1}\right)$, as shown by Figure 3.

I actually computed the general formula for $\left(x_{1}, y_{1}\right)$ in terms of $x_{0}, y_{0}, a, b, c, d$. The expression for $x_{1}$ involves about 100 monomials, so I do not reproduce it here. Such a result seems nearly useless for most purposes. A few years ago I would have dismissed it too. Nevertheless, I would like to emphasize that symbolic manipulation programs have no difficulty handling polynomials of degree several hundred, so computationally these formulas can be quite useful.

For $m \geq 5$ one can try variants of the above tricks to get new solutions out of old ones, but no general method was ever found. In fact, there can be no such method as shown by the following theorem of Faltings83.

Theorem 9. If $g(x, y)=0$ defines a curve of genus $\geq 2$, then $g(x, y)=0$ has only finitely many rational solutions. In particular, if $m \geq 5$ and $f_{m}$ has no multiple roots, then $y^{2}=f_{m}(x)$ has only finitely many rational solutions.

10 (Differential forms without poles). Already Euler was aware of the curious property of

$$
\int \frac{d x}{\sqrt{x^{3}+a x^{2}+b x+c}}
$$

that the value of the integral on any curve in the complex domain is finite. He also knew that the integrals

$$
\int \frac{p(x)}{q(x)} d x \quad \text { and } \quad \int \frac{P\left(x, \sqrt{x^{2}+b x+c}\right)}{Q\left(x, \sqrt{x^{2}+b x+c}\right)} d x
$$

where $p(x), q(x), P(x, y), Q(x, y)$ are polynomials never have this property. Indeed, integrals of rational functions diverge near the poles, and if there are no poles, then they diverge near infinity. The seemingly more complicated second case can be 
reduced to the first one by a suitable substitution $x=X(t), y=Y(t)$ where $X, Y$ are rational functions. (In fact, these are exactly the coordinates of the inverse function of the stereographic projection of the conic $\left(y^{2}=x^{2}+b x+c\right)$ from one of its points.)

It is somewhat annoying that the integral [10,1) is 2-valued, and Riemann suggested the following way to correct this.

Instead of looking at [10,1) as an integral over the $x$-axis, we look at this as an integral over the curve $C=\left(y^{2}=x^{3}+a x^{2}+b x+c\right)$. This has the great advantage that

$$
\left.\sqrt{x^{3}+a x^{2}+b x+c}\right|_{C}=\left.y\right|_{C}
$$

hence we get the much simpler looking expression

$$
\int \frac{d x}{y}, \quad \text { where we integrate over a path in } C \text {. }
$$

It seems now that this integral should diverge where $y=0$, that is, at the roots of $x^{3}+a x^{2}+b x+c$. However, we integrate over $y^{2}=x^{3}+a x^{2}+b x+c$; thus

$$
\left.2 y d y\right|_{C}=\left.\left(3 x^{2}+2 a x+b\right) d x\right|_{C},
$$

and so

$$
\int_{\Gamma \subset C} \frac{d x}{y}=\int_{\Gamma \subset C} \frac{2 d y}{3 x^{2}+2 a x+b}
$$

Since $x^{3}+a x^{2}+b x+c$ has no multiple roots, $3 x^{2}+2 a x+b$ is not zero at the roots, so either $y$ or $3 x^{2}+2 a x+b$ is nonzero for every point $(x, y) \in C$.

Near infinity the integrand grows like $x^{-\frac{3}{2}}$; hence there we have convergence.

A generalization of this observation to the higher degree cases is given by the following theorem which was already known to Abel.

Theorem 11. Let $f(x)$ be a polynomial of degree $m$ without multiple roots and $\Phi(x, y)$ a meromorphic function on $\mathbb{C}^{2}$. The integral

$$
\int \Phi(x, \sqrt{f(x)}) d x
$$

is finite on every path $\Gamma \subset\left(y^{2}=f(x)\right)$ iff it is identical to

$$
\int \frac{p(x)}{\sqrt{f(x)}} d x
$$

where $p$ is a polynomial of degree $\leq \frac{m-3}{2}$.

In particular, the number of linearly independent integrals with this finiteness property is precisely the genus of the curve $y^{2}=f(x)$.

12 (Summary). The results explained above all generalize to arbitrary plane curves, and even to algebraic space curves. I formulate them in the genus zero case, since this is the one that we would like to understand in higher dimensions.

First we need some definitions.

Definition 13. We start with some polynomials

$$
f_{1}\left(x_{1}, \ldots, x_{n}\right), \ldots, f_{k}\left(x_{1}, \ldots, x_{n}\right)
$$


in $n$ (complex) variables. Viewed as a system of polynomial equations, their common zero set is the affine algebraic variety

$$
X=\left(f_{1}\left(x_{1}, \ldots, x_{n}\right)=\cdots=f_{k}\left(x_{1}, \ldots, x_{n}\right)=0\right) .
$$

Affine refers to the circumstance that we look at solutions in affine $n$-space $\mathbb{A}^{n}$. We can always look at all complex solutions, denoted by $X(\mathbb{C})$. If the $f_{i}$ have real coefficients, then it is sensible to consider all real solutions, denoted by $X(\mathbb{R})$. If the $f_{i}$ have rational coefficients, then we have a system of Diophantine equations and the set of rational solutions is denoted by $X(\mathbb{Q})$.

A rational map from $\mathbb{A}^{n}$ (with coordinates $x_{1}, \ldots, x_{n}$ ) to $\mathbb{A}^{m}$ (with coordinates $\left.y_{1}, \ldots, y_{m}\right)$ is given by $m$ rational functions

$$
\Phi:\left(x_{1}, \ldots, x_{n}\right) \mapsto\left(\phi_{1}(\mathbf{x}), \ldots, \phi_{m}(\mathbf{x})\right) .
$$

Note that $\Phi$ may not be everywhere defined. We say that $\Phi$ is a morphism if it is everywhere defined. If $X \subset \mathbb{A}^{n}$ and $Y \subset \mathbb{A}^{m}$ are varieties and $\Phi(X) \subset Y$, then we say that $\Phi$ gives a rational map of $X$ to $Y$.

In what follows, I make two simplifying assumptions. It is known that these do not result in any loss of generality.

1. $X$ is smooth. This is equivalent to assuming that $X(\mathbb{C}) \subset \mathbb{C}^{n} \cong \mathbb{R}^{2 n}$ is a differentiable submanifold.

2. $X$ is irreducible. This is equivalent to assuming that $X(\mathbb{C})$ is connected.

As a topological space, $X(\mathbb{C})$ is always even dimensional, and we define the (complex) dimension $\operatorname{dim} X$ of $X$ to be one half of the (real) topological dimension of $X(\mathbb{C})$. Thus $\operatorname{dim} \mathbb{C}^{n}=n$. It is easy to see that $X(\mathbb{R})$ is either empty or of (real) dimension $\operatorname{dim} X$.

$X$ is a curve, surface, 3-fold, etc., if $\operatorname{dim} X$ is $1,2,3$, etc.

Already in the curve case we saw that it is convenient to throw in some points at infinity. This can be done for any variety $X$, though the resulting compactification is not unique in dimensions $\geq 2$. For now this ambiguity does not matter; any of these will be denoted by $\bar{X}$.

Theorem 14. Let

$$
X=\left(f_{1}\left(x_{1}, \ldots, x_{n}\right)=\cdots=f_{k}\left(x_{1}, \ldots, x_{n}\right)=0\right)
$$

be a (smooth and irreducible) algebraic curve. The following are equivalent.

1. $\bar{X}(\mathbb{C})$ is homeomorphic to $S^{2}$.

2. There are rational functions $h_{1}(t), \ldots, h_{n}(t)$ such that

$$
t \mapsto\left(h_{1}(t), \ldots, h_{n}(t)\right)
$$

is a one-to-one map from the Riemann sphere to $\bar{X}(\mathbb{C})$.

3. Let $\Phi_{1}(\mathbf{x}), \ldots, \Phi_{n}(\mathbf{x})$ be arbitrary meromorphic functions on $\mathbb{C}^{n}$. Then

$$
\int \Phi_{1}(\mathbf{x}) d x_{1}+\cdots+\Phi_{n}(\mathbf{x}) d x_{n}
$$

diverges along some path in $X(\mathbb{C})$.

If the $f_{i}$ have real coefficients, then (1-3) imply:

4. $X(\mathbb{R})$ is either empty or $S^{1}$.

If the $f_{i}$ have rational coefficients, then (1-3) imply:

5. If the system of equations $f_{1}(\mathbf{x})=\cdots=f_{k}(\mathbf{x})=0$ has a rational solution, then it has infinitely many. 
6. The system of equations $f_{1}(\mathbf{x})=\cdots=f_{k}(\mathbf{x})=0$ always has a solution in a field $\mathbb{Q}(\sqrt{d})$ for some $d \in \mathbb{Q}$.

It may be a little surprising, but in practice condition (14.3) is the easiest to verify. This is especially so with its higher dimensional analogs.

It is possible to formulate the last 2 assertions so that they become equivalent to $(141-3)$.

7. The system of equations $f_{1}(\mathbf{x})=\cdots=f_{k}(\mathbf{x})=0$ has a sequence of solutions $\left(x_{i 1}, \ldots, x_{i n}\right)$ in a field $\mathbb{Q}(\sqrt{d})$ where the denominators and numerators of the $x_{i j}$ grow polynomially in $i$.

Definition 15. An algebraic curve $X$ is called geometrically rational if it satisfies the equivalent conditions $(141-3)$.

Many authors simply refer to these as rational curves. This is one of the standard terminological sources of confusion in algebraic geometry.

Let me also emphasize that while a real algebraic variety is a variety defined by real equations, a rational curve (or variety) has nothing to do with its equations having rational coefficients.

Theorem 16. Let $k$ be a field with algebraic closure $\bar{k}$ and

$$
X=\left(f_{1}\left(x_{1}, \ldots, x_{n}\right)=\cdots=f_{m}\left(x_{1}, \ldots, x_{n}\right)=0\right)
$$

be a (smooth and irreducible) algebraic curve over $k$. The following are equivalent.

1. $\bar{X}$ is isomorphic to $\mathbb{P}^{1}$ over $\bar{k}$.

2. There is a plane conic $Q=(q(s, t)=0)$ defined over $k$ and rational functions $h_{1}(s, t), \ldots, h_{n}(s, t)$ (with coefficients in $k$ ) such that

$$
Q \ni(s, t) \mapsto\left(h_{1}(s, t), \ldots, h_{n}(s, t)\right) \in X
$$

is an isomorphism from the conic $\bar{Q}$ to $\bar{X}$.

\section{RAtional SURFACES}

After the successful division of curves into 3 broad classes, one should naturally attempt a similar classification for surfaces. This turns out to be a much bigger undertaking, and instead I would like to focus on generalizing the detailed description of rational curves obtained in theorems (14) and (16):

Find the 2-dimensional analogs of rational curves.

Keep in mind that we want to do at least two different things at the same time. The easier part is to classify all algebraic surfaces over $\mathbb{C}$ that behave like $\mathbb{C}^{2}$. The second, harder, part is to perform a similar classification over any field. The difference between these is illustrated by quadrics. In order to streamline the discussion, we need to say a few words about projective varieties.

Definition 17. In dimension 2 and up it is harder and harder to ignore the precise compactification $\bar{X}$ of a variety $X$. The natural compactification of $\mathbb{C}^{n}$ is the projective space $\mathbb{C P}^{n}$. As a set this is the collection of nonzero $n+1$-tuples $\left(x_{0}\right.$ : $\cdots: x_{n}$ ) modulo the equivalence relation

$$
\left(x_{0}: \cdots: x_{n}\right) \sim\left(c x_{0}: \cdots: c x_{n}\right) \quad \text { for } 0 \neq c \in \mathbb{C} .
$$

Despite this ambiguity, the zero set of a homogeneous polynomial is still well defined. Thus, proceeding as in (13) we end up with projective varieties. 
Example 18 (Quadrics in $\mathbb{P}^{3}$ ).

Every smooth quadric surface $Q \subset \mathbb{P}^{3}$ is given by a diagonal equation

$$
a_{0} x_{0}^{2}+\cdots+a_{3} x_{3}^{2}=0 \quad \text { with } \quad a_{0} \cdots a_{3} \neq 0
$$

(except in characteristic 2). Further simplifications of the equation depend on the field:

1. Over $\mathbb{C}$ the substitutions $y_{i}=\sqrt{a_{i}} x_{i}$ give the equation

$$
y_{0}^{2}+\cdots+y_{3}^{2}=0 .
$$

2. Over $\mathbb{R}$ the substitutions $y_{i}=\sqrt{\left|a_{i}\right|} x_{i}$ give an equation

$$
\pm y_{0}^{2} \pm \cdots \pm y_{3}^{2}=0 \text {, }
$$

giving 3 types up to isomorphisms.

3. Over $\mathbb{Q}$ there are many more cases. For instance, if the $p_{i}$ are different primes, then the isomorphism class of the quadric

$$
Q\left(p_{1}, \ldots, p_{k}\right):=\left(x_{0}^{2}-x_{1}^{2}+x_{2}^{2}-\prod_{i} p_{i} x_{3}^{2}=0\right)
$$

determines the primes $p_{1}, \ldots, p_{k}$. (This can be seen, for instance, by noting that the discriminant of a quadratic form changes by a square under a coordinate change.)

Although the quadrics $Q\left(p_{1}, \ldots, p_{k}\right)$ are pairwise nonisomorphic, they all are very much like $\mathbb{P}^{2}$. Noting that $P=(1: 1: 0: 0)$ is a solution of each of them, let us project $Q\left(p_{1}, \ldots, p_{k}\right)$ from $P$ to the $\left(x_{0}=0\right)$ plane:

$$
\pi:\left(x_{0}: x_{1}: x_{2}: x_{3}\right) \mapsto\left(\frac{x_{1}-x_{0}-x_{3}}{x_{3}}+1: \frac{x_{2}}{x_{3}}: 1\right) .
$$

The inverse is

$$
(u+1: v: 1) \mapsto(1-\lambda: 1+\lambda u: \lambda v: \lambda) \quad \lambda=\frac{2(1+u)}{1-u^{2}+v^{2}-\prod_{i} p_{i}} .
$$

Notice, however, that strictly speaking $\pi$ and $\pi^{-1}$ are not inverses of each other. Indeed, $\pi$ is not defined at $P$, and two lines $L_{ \pm}$in $Q\left(p_{1}, \ldots, p_{k}\right)$ are mapped to points

$$
\pi:\left(1: 1: \pm t \sqrt{\prod_{i} p_{i}}: t\right) \rightarrow\left(0: \pm \sqrt{\prod_{i} p_{i}}: 1\right) .
$$

Similarly, $\pi^{-1}$ is not defined at the points $\left(0: \pm \sqrt{\prod_{i} p_{i}}: 1\right)$, and the line $M=$ $(0: u: 1)$ is mapped to the point $P$. Thus the best we can say is that

$$
\begin{aligned}
& \pi \text { and } \pi^{-1} \text { give isomorphisms between the } \\
& \text { open sets } Q \backslash L_{ \pm} \subset Q \text { and } \mathbb{P}^{2} \backslash M \subset \mathbb{P}^{2} \text {. }
\end{aligned}
$$

From the point of view of diophantine problems this is entirely satisfactory. We get a complete description of the rational points of $Q$ which lie outside $L_{ \pm}$. The remaining question of describing all rational points on $L_{ \pm}$is a lower dimensional one and very easy in this case.

The situation is not so clear topologically. It is not straightforward to use $\pi$ to get good information about the topology of the complex or real points of $Q$. We will see, however, that similar ideas can be used very effectively.

For now we establish a general definition. 
Definition 19. Let $X$ and $Y$ be projective varieties. We say that $X$ and $Y$ are birational if there are dense open sets $X^{0} \subset X$ and $Y^{0} \subset Y$ such that $X^{0}$ and $Y^{0}$ are isomorphic (as algebraic varieties).

If $X$ and $Y$ are defined over a field $k$ (for instance $\mathbb{Q}$ or $\mathbb{R}$ ), then we insist that $X^{0}, Y^{0}$ and the isomorphism $X^{0} \cong Y^{0}$ be defined by polynomials with coefficients in that field.

If $K \supset k$ is a field extension, we say that $X$ and $Y$ are birational over $K$ if we want to use $K$ as the coefficient field.

Definition 20. Let $X$ be a variety defined over a field $k$. We say that $X$ is rational if $X$ is birational to $\mathbb{P}^{\operatorname{dim} X}$. As above we can define the notion rational over $K$ if we want to use a larger field $K \supset k$ as the coefficient field.

In many diophantine problems, rationality is the ideal state of affairs. If $X$ is rational over $\mathbb{Q}$, then we understand all the $\mathbb{Q}$-points in a dense subset $X^{0}$. The remaining questions about $X \backslash X^{0}$ pose a lower dimensional problem.

With these definitions in mind, we are ready to try to classify all algebraic surfaces which behave like rational curves. The main claim is summarized in the following thesis:

Over $\mathbb{C}$, the correct 2-dimensional analog of rational curves is the class of rational surfaces.

21 (Supporting evidence). In dimension 1, the simple topological characterization of rational curves is very appealing. There is no similarly simple topological characterization of rational surfaces. In fact, this turned out to be a rather subtle question. There are nonrational surfaces which are homeomorphic to a rational surface Dolgachev66. In the $C^{\infty}$-setting the question was settled by Donaldson's theory of differentiable 4 -manifolds:

1. Let $X$ be a smooth projective surface over $\mathbb{C}$ such that $X(\mathbb{C})$ is diffeomorphic to a rational surface. Then $X$ is also rational.

A characterization using convergent integrals also gets more complicated. First of all, we need to use both line integrals and surface integrals

$$
\int \sum_{i} \Phi_{i}(\mathbf{x}) d x_{i} \text { and } \int \sum_{i j} \Phi_{i j}(\mathbf{x}) d x_{i} \wedge d x_{j} .
$$

Furthermore, in the surface integral case we have to allow $\Phi_{i j}$ to be a 2 -valued holomorphic function (that is, locally like the square root of a holomorphic function).

2. A smooth projective surface $X \subset \mathbb{C}^{n}$ is rational iff every line integral diverges along some path and every 2 -valued holomorphic surface integral diverges along some surface.

The question of saying something about the real topology $X(\mathbb{R})$ is left till (49)).

The surface analogs of (16) are more complicated. We start by describing some examples.

22 (Easy examples).

The projective plane $\mathbb{P}^{2}$ and quadrics $Q \subset \mathbb{P}^{3}$.

Stereographic projection from any point of the quadric as in (8) shows that quadrics in any dimension behave very much like plane conics.

(There is one technical point here that should be mentioned. There are many algebraic surfaces over $\mathbb{Q}$ which are isomorphic to $\mathbb{P}^{2}$ over $\mathbb{C}$ but not over $\mathbb{Q}$. It turns 
out that these never have any rational points and they are rather easy to understand using Galois cohomology (cf. [Serre79]). It is rather awkward to describe them by equations, so I will ignore them in the sequel. Fortunately, similar problems do not appear with the other examples.)

Next we get to the most famous example of surface theory:

23 (Cubic surfaces). These are smooth surfaces $S \subset \mathbb{P}^{3}$ defined by a single cubic equation.

At first these seem unlikely to behave like rational curves since degree 3 plane curves are not rational. Nonetheless, we see below that cubics in 4 variables are simpler than cubics in 3 variables.

Let us first look at the equation

$$
T:=\left(x^{2} y+y^{2} z+z^{2} v+v^{2} x=0\right) \subset \mathbb{P}^{3} .
$$

The surface $T$ contains several lines; for instance

$$
L_{1}=(t: 0: 1: 0) \quad \text { and } \quad L_{2}=(0: s: 0: 1)
$$

are skew lines on $T$. The line connecting $P_{1}(t)=(t: 0: 1: 0)$ and $P_{2}(s)=$ $(0: s: 0: 1)$ intersects $T$ in one more point,

$$
P(s, t)=\left(t\left(s^{2}+t\right): s\left(s t^{2}+1\right): s^{2}+t: s t^{2}+1\right) .
$$

It is easy to see that

$$
\mathbb{A}^{2} \rightarrow T: \quad(s, t) \mapsto P(s, t)
$$

is a birational map between the plane and the points of $T$.

One can prove that every cubic surface over $\mathbb{C}$ contains a pair of skew lines. This leads to the first substantial result in the birational geometry of surfaces:

Theorem 24. Clebsch1866 A smooth cubic surface over $\mathbb{C}$ is always rational.

The situation is more complicated over other fields. As another example, let us look at the cubics

$$
S_{a}:=\left(x^{3}+y^{3}+z^{3}=a v^{3}\right) \subset \mathbb{P}^{3} .
$$

There are plenty of pairs of skew complex lines in $S_{a}$, but is easy to check that $S_{a}$ does not contain a pair of skew real lines.

If $a \in \mathbb{Q}$ and we want to work over $\mathbb{Q}$, the problem becomes even more complicated. The answer is, however, completely known due to the following beautiful theorem:

Theorem 25. Segre51 Assume that $a \in \mathbb{Q}$. Then $S_{a}$ is rational (over $\mathbb{Q}$ ) iff $a$ is a cube.

If $a=b^{3}$, then $v \mapsto b^{-1} v$ reduces us to the case $a=1$. This case is indeed rational, as shown by the next result which I state in two equivalent forms: 
Proposition 26. Let $S_{1}$ be the cubic surface $\left(x^{3}+y^{3}+z^{3}=v^{3}\right) \subset \mathbb{P}^{3}$ and $\Phi$ : $(s, t) \mapsto(x, y, z)$ the map given by

$$
\begin{aligned}
x & =-\frac{1}{3} \frac{t^{4}+3 t^{2} s^{2}-2 t^{3} s-2 t s^{3}+s^{4}+9 t}{2 t^{2} s-2 t s^{2}-t^{3}+s^{3}+3} \\
y & =\frac{1}{3} \frac{t^{4}+3 t^{2} s^{2}-2 t^{3} s-2 t s^{3}+s^{4}+9 s-9 t}{2 t^{2} s-2 t s^{2}-t^{3}+s^{3}+3} \\
z & =\frac{-t^{2} s+t s^{2}+t^{3}+3}{2 t^{2} s-2 t s^{2}-t^{3}+s^{3}+3} .
\end{aligned}
$$

Then

1. $\Phi$ gives a birational map $\mathbb{A}^{2} \rightarrow S_{1}$ via $(s, t) \mapsto(x: y: z: 1)$.

2. The general rational solution of $x^{3}+y^{3}+z^{3}=1$ can be uniquely written as $\Phi(s, t)$ for some rational $s, t$.

27 (Explanation of the formulas). We started with the conjugate pair of lines $L_{i}=$ $\left(t:-\epsilon_{i} t: \epsilon_{i}: 1\right)$ in $S_{1}$ where $\epsilon_{i}$ for $i=1,2$ are the complex cube roots of 1 . As in (23) we obtain a birational map $L_{1} \times L_{2}-\rightarrow S_{1}$.

We obtain another birational map $\mathbb{A}^{2} \rightarrow L_{1} \times L_{2}$ by choosing the line pair $\left(t, \epsilon_{i} t\right)$ as the coordinates in $\mathbb{A}^{2}$.

Both of these maps are defined only over $\mathbb{Q}\left(\epsilon_{i}\right)$, but the composite, our $\Phi$, is defined over $\mathbb{Q}$. (This is not an accident but a consequence of the theory of Galois descent; cf. Serre79].) The actual computations were carried out by Maple.

Most cubics are not rational over $\mathbb{Q}$, but in many cases there is a map $\Phi: \mathbb{P}^{2}--\rightarrow S$ defined over $\mathbb{Q}$ which is nondegenerate, meaning that the image of $\Phi$ does not lie in any curve. This notion is quite useful in general:

Definition 28. A variety $X$ of dimension $n$ is unirational if there is a rational map $\Phi: \mathbb{P}^{n} \rightarrow X$ whose image is not contained in any smaller closed algebraic subset.

As before, if $X$ is defined over a field $k$, then we want $\Phi$ to be defined over $k$ as well.

Let $\Phi: \mathbb{P}^{n} \rightarrow X$ be a map whose image is not contained in any smaller closed algebraic subset. It is good to get an idea how big $\Phi\left(\mathbb{P}^{n}\right)$ is.

1. $\Phi\left(\mathbb{C P}^{n}\right)$ contains a dense open subset of $X(\mathbb{C})$.

2. $\Phi\left(\mathbb{R P}^{n}\right)$ contains an open subset of $X(\mathbb{R})$, but it may not be dense.

3. $\Phi\left(\mathbb{Q P}^{n}\right)$ may be a rather sparse subset of $X(\mathbb{Q})$.

These possibilities are illustrated by the example

$$
\Phi: \mathbb{A}^{1} \rightarrow \mathbb{A}^{1} \quad \Phi(x)=x^{2} .
$$

Despite these shortcomings unirationality is an important and useful notion.

Theorem 29. Segre42 Let $S \subset \mathbb{P}^{3}$ be a smooth cubic surface defined by an equation $(F=0)$ with rational coefficients. Then $S$ is unirational over $\mathbb{Q}$ iff $S$ has at least one rational point.

The proof of Segre works over any field of characteristic zero and also for higher dimensional cubics satisfying a certain genericity assumption. It is, however, only recently that the result has been extended to all cubics and to all fields. (The key missing cases were finite fields.)

Theorem 30. Kollár00b] Let $k$ be a field, $n \geq 3$ and $X \subset \mathbb{P}^{n}$ a smooth cubic hypersurface over $k$. Then $X$ is unirational over $k$ iff $X$ has a $k$-point. 
We go through the other "rational like" examples at a faster pace.

31 (Relatives of cubics). There are two other classes of equations which have been studied classically and which behave very much like cubic surfaces. These are

1. (Quartic double planes) These can be given as

$$
x^{2}=f(y, z) \subset \mathbb{A}^{3} \quad \text { where } \operatorname{deg} f=4 .
$$

2. (Intersections of 2 quadrics) Defined by 2 quadratic equations

$$
Q_{1}(x, y, z, t)=Q_{2}(x, y, z, t)=0 \subset \mathbb{A}^{4} .
$$

The basic theory of these is very similar to that of cubic surfaces.

The following is a much larger class, though less studied classically.

32 (Conic bundles). These are surfaces which can be given by an equation which is quadratic in 2 of the variables. As a typical example consider

$$
(q(x, y)=f(z)) \subset \mathbb{A}^{3} \quad \text { where } q \text { is a quadric. }
$$

In analogy with the curve case it would seem that these are rational only if $f$ has low degree. The appearance of 2 quadratic variables, however, changes the situation completely. Indeed, over $\mathbb{C}$ by a change of the $x, y$ variables we can assume that $q(x, y)=x^{2}-y^{2}$. Then

$$
(u, v) \mapsto\left(\frac{f(v)+u^{2}}{2 u}, \frac{f(v)-u^{2}}{2 u}, v\right)
$$

gives a rational map between the plane and the points of $x^{2}-y^{2}=f(z)$.

For other cases of $q(x, y)$ the situation is quite different. For instance, $x^{2}+y^{2}=$ $f(z)$ is rational over $\mathbb{R}$ iff $f$ has at most 2 real roots of odd multiplicity. This is rather easy to see topologically. Assume for simplicity that there are no multiple roots. If $f$ has $2 d$ real roots, then $f$ is positive on $d$ disjoint intervals and so the real points of $x^{2}+y^{2}=f(z)$ consist of $d$ connected components. It is easy to see (though not quite obvious) that the number of connected components of $X(\mathbb{R})$ is a birational invariant over $\mathbb{R}$ (for smooth varieties).

There are a few more examples which do not fit neatly into the above patterns. They also do not appear very frequently.

33 (Esoteric examples).

1. Surfaces defined by equations

$$
x^{2}+y^{3}+y g_{4}(z)+g_{6}(z)=0
$$

where $\operatorname{deg} g_{i} \leq i$. These are quite a bit more complicated than quartic double planes.

2. Let $M$ be a $5 \times 5$ matrix whose entries are linear forms in 6 variables. The skew-symmetric $4 \times 4$ subdeterminants are degree 4 polynomials, which are squares $Q_{1}^{2}, \ldots, Q_{5}^{2}$. The equations

$$
\left(Q_{1}=\cdots=Q_{5}=0\right) \subset \mathbb{P}^{5}
$$

define a rational surface. These surfaces are actually quite easy to understand.

3. Compactifications of homogeneous spaces under the group $G L(1) \times G L(1)$. Here the general methods of group cohomology work very well. 
It is not at all clear, but we ran out of examples. This is the main theorem of the theory of rational surfaces over any field which will be given in a much stronger form in the next section.

\section{Minimal models}

As we have seen, it is quite reasonable to study algebraic varieties up to birational equivalence. This raises the question:

Is there a particularly simple variety in every birational equivalence class? If yes, how can we find it?

We start answering these questions by first finding ways to make a variety more complicated.

Definition 34 (Blowing up).

Let $X$ be a smooth variety and $x \in X$ a point. Embed $X$ in a large projective space $X \subset \mathbb{P}^{N}$ such that no 3 of its points are on a line. (As usual in algebraic geometry, we also assume the degenerate versions of this. That is, no secant line is tangent and there are no inflection tangents.)

For $p \in \mathbb{P}^{N}$ let $\pi_{p}: \mathbb{P}^{N} \rightarrow \mathbb{P}^{N-1}$ denote the projection from $p$.

If $p \in \mathbb{P}^{N}$ is outside $X$, then $\pi_{p}: X \rightarrow \pi_{p}(X)$ is an isomorphism. If $p \in X$, then

$$
\pi_{p}: X \backslash\{x\} \rightarrow \pi_{p}(X \backslash\{x\})
$$

is still an isomorphism, but the closure of $\pi_{p}(X \backslash\{x\})$ is bigger than $X$. To be precise, the point $p$ is replaced by all the tangent directions of $X$ at $p$.

That is, given $X$ of dimension $n$ and a point $x \in X$, we constructed a variety $B_{x} X$ such that

$$
B_{x} X=(X \backslash\{x\}) \cup \mathbb{P}^{n-1} .
$$

It turns out that this construction does not depend on the embedding $X \subset \mathbb{P}^{N}$. $X$ and $B_{x} X$ are clearly birational, and it is quite reasonable to say that $B_{x} X$ is "more complicated" than $X$.

The natural map $B_{x} X \rightarrow X$ is everywhere defined. This map, or $B_{x} X$ itself, is called the blow up of $x \in X$.

35 (The topology of blow ups). We compute a local model for blow ups.

Let $0 \in \mathbb{C}^{n}$ be the origin and $B \subset \mathbb{C}^{n}$ the unit ball. Let $\pi: B_{0} \mathbb{C}^{n} \rightarrow \mathbb{C}^{n}$ be the blow up of the origin. We would like to understand $\pi^{-1}(B)$. By our construction, this looks like

$$
(B \backslash\{0\}) \cup(\text { a point for every line through } 0) .
$$

Inversion on the unit sphere shows that $\mathbb{C}^{n} \backslash \bar{B}$ is diffeomorphic to $B \backslash\{0\}$ and the hyperplane

$$
\mathbb{C P}^{n-1} \cong H=\mathbb{C P}^{n} \backslash \mathbb{C}^{n} \subset \mathbb{C P}^{n}
$$

has one point for every line through 0 . Thus it is a reasonable guess that $\pi^{-1}(B)$ is diffeomorphic to $\mathbb{C P}^{n} \backslash \bar{B}$. This is indeed true, but this diffeomorphism reverses orientation. We let $\overline{\mathbb{C P}}^{n}$ denote $\mathbb{C P}^{n}$ with opposite orientation. (This is a bit unfair since I have not told you what the "standard" orientation of $\mathbb{C P}^{n}$ is, but luckily this will not matter in these lectures.)

The same argument applies over $\mathbb{R}$, but here the orientation reversal does not matter. (In fact $\mathbb{R P}^{n}$ is not orientable for $n$ even.) Thus we obtain: 
Proposition 36. Let $X$ be a smooth complex variety and $x \in X$ a point. Then

$$
B_{x} X(\mathbb{C}) \text { is diffeomorphic to } X(\mathbb{C}) \# \overline{\mathbb{C P}}^{n} \text {, }
$$

where \# denotes the connected sum operation. If $X$ is a real variety and $x \in X$ is a real point, then

$$
B_{x} X(\mathbb{R}) \text { is diffeomorphic to } X(\mathbb{R}) \# \mathbb{R} \mathbb{P}^{n} .
$$

Definition 37 (Minimal models of surfaces). Let $S$ be a smooth projective surface. In trying to find simple birational models of $S$, first we want to undo all blow ups. That is, if $S$ is isomorphic to $B_{x} S_{1}$ for some $S_{1}$, then we replace $S$ by $S_{1}$ and continue. Thus we get a sequence of contractions

$$
S \rightarrow S_{1} \rightarrow \cdots \rightarrow S_{k}=S^{*}
$$

where $S^{*}$ is not the blow up of anything else. (There are many ways to see that the process will stop. For instance, the second Betti number of the complex points drops by 1 at each step.) $S^{*}$ is called a minimal model of $S$.

A basic result of the birational geometry of surfaces (cf. [BPV84]) asserts that a minimal model is almost always unique.

Theorem 38. Let $S$ be a smooth projective surface over $\mathbb{C}$.

1. If $S$ is not birational to $\mathbb{P}^{1} \times($ curve), then $S$ has a unique minimal model.

2. If $S$ is birational to $\mathbb{P}^{1} \times($ curve), then every minimal model of $S$ is either

(a) $\mathbb{P}^{2}$, or

(b) a $\mathbb{P}^{1}$-bundle over a curve.

If a surface $S$ is defined over a field $k$, then we are especially interested in birational maps that are defined over $k$. First we have to see which blow ups make sense over $k$. Let us look for instance at the case $k=\mathbb{R}$.

It is clear that we can blow up a real point $p \in S(\mathbb{R})$ and $B_{x} S$ is still defined by real equations. It may be a little less clear that if $p \in S(\mathbb{C})$ is a complex point with conjugate $\bar{p} \in S(\mathbb{C})$, then the 2 point blow up $B_{p, \bar{p}} S$ is again definable by real equations.

Similarly, for any field $k$ we can blow up either points in $S(k)$ or Galois invariant finite subsets of $S(\bar{k})$.

This leads to an analog of the minimal model theory over any fields, giving the following result which was gradually developed by Castelnuovo, Comessatti, Segre, Iskovskikh and Mori.

Theorem 39. Let $S$ be a smooth projective surface over any field $k$.

1. If $S_{\bar{k}}$ is not birational to $\mathbb{P}^{1} \times($ curve), then $S$ has a unique minimal model over $k$.

2. If $S_{\bar{k}}$ is rational, then every minimal model of $S$ is among those listed in (22) -(33). (I am cheating again since I have not fully described the projective versions of several of these examples.)

\section{Rationally CONNECTED VARIETIES}

Already in the surface case it is not easy to show that all low degree surfaces are rational. Therefore it did not come as a big surprise that in higher dimensions rational varieties are too special. By now we should expect that a cubic hypersurface $X_{3}^{n} \subset \mathbb{P}^{n+1}$ is analogous to rational curves. M. Noether knew that every smooth 
cubic hypersurface of dimension at least 2 is unirational over $\mathbb{C}$, but nobody was able to prove that $X_{3}^{n}$ is rational for $n \geq 3$. (And indeed, smooth cubic 3-folds are not rational by ClemensGriffiths72.)

Unfortunately, it seems that the class of unirational varieties is still too restrictive. For instance, extrapolating from plane conics and cubic surfaces, we should expect that hypersurfaces $X \subset \mathbb{P}^{n}$ behave analogously to rational curves iff $\operatorname{deg} X \leq n$. (There are also much better reasons to believe this.)

Morin40 proved that a degree $d$ hypersurface in $\mathbb{P}^{n}$ is unirational if $d$ is very small (less that an iterated logarithm of $n$ ), but the general case seems hopeless. The smallest unknown example is degree 4 hypersurfaces in $\mathbb{P}^{4}$. In fact, it is believed that a general degree 4 hypersurface in $\mathbb{P}^{4}$ is not unirational.

To remedy the situation, a new concept was proposed in KoMiMo92. Instead of trying to emulate global properties of $\mathbb{C P}^{n}$, we concentrate on rational curves. $\mathbb{C P}^{n}$ has lots of rational curves (lines, conics and many higher degree ones), all of which are images of maps $\mathbb{C P}^{1} \rightarrow \mathbb{C P}^{n}$. This leads us to the following informal definition.

A variety $X$ is rationally connected iff there are plenty of rational curves on $X$.

The main thesis of [KoMiMo92] is that the above definition is the right one:

Rationally connected varieties are the correct higher dimensional analogs of rational curves.

Before we can even start arguing the above thesis, the informal definition has to be made precise.

There are several a priori sensible ways of defining what "plenty of rational curves" should mean. Fortunately, many of these are equivalent. The equivalence of the various versions was the first strong evidence that the proposed definition is interesting.

Theorem 40. KoMiMo92, Kollár98a Let $X$ be a smooth projective variety over C. The following are equivalent:

1. There is a rational curve through any 2 points of $X$.

2. There is an open subset $\emptyset \neq X^{0} \subset X$ such that there is a rational curve through any 2 points of $X^{0}$.

3. There is a rational curve through any (finite) number of points of $X$.

4. Let $p_{1}, \ldots, p_{n} \in \mathbb{C P}^{1}$ be distinct points. For each $i$ let $f_{i}: D\left(p_{i}\right) \rightarrow X(\mathbb{C})$ be a holomorphic map from a small disc around $p_{i}$ to $X(\mathbb{C})$. Let $n_{i}$ be natural numbers. Then there is a morphism $f: \mathbb{C P}^{1} \rightarrow X$ such that the Taylor series of $f_{i}$ and of $f \mid D\left(p_{i}\right)$ coincide up to order $n_{i}$ for every $i$.

5. There is a morphism $f: \mathbb{C P}^{1} \rightarrow X$ such that $f^{*} T_{X}$ is ample. (Over $\mathbb{P}^{1}$ this is equivalent to being a direct sum of positive degree line bundles).

Now we can make our definition precise:

Definition 41. A smooth projective variety $X$ over $\mathbb{C}$ is called rationally connected if it satisfies the equivalent properties in (40).

The class of rationally connected varieties is stable under many operations:

1. If $X$ is birational to a rationally connected variety, then $X$ itself is rationally connected. This easily follows from [40,2). 
2. More generally, the (closure of the) image of a rationally connected variety is rationally connected.

3. A smooth hypersurface $X \subset \mathbb{P}^{n}$ is rationally connected iff $\operatorname{deg} X \leq n$.

Rational connectedness also behaves well in families. This was implicit in our earlier results on surfaces. Once the shape of the equation was specified, the actual coefficients did not matter in deciding rationality over $\mathbb{C}$. Being rational is probably not deformation invariant in dimensions 3 and up, but rational connectedness is:

Theorem 42. KoMiMo92 Let $X_{t}: t \in[0,1]$ be a continuously varying family of smooth varieties. If $X_{0}$ is rationally connected, then so is $X_{1}$.

Ideally one would like an even stronger form of this result. The spaces $X_{t}(\mathbb{C})$ are all diffeomorphic, so (42) would be a consequence of a topological characterization of rationally connected varieties. It turns out that diffeomorphism alone does not characterize rationally connected varieties. One has to look at the symplectic structure as well. In the symplectic setting we get a conjectural analog of (21), but it is not even known in dimension 3. See Kollár98a for details.

A characterization via convergent integrals is also interesting. It turns out that such an $X$ does not carry holomorphic differential forms of any kind:

Theorem 43. KoMiMo92 Let $X$ be a smooth, projective, rationally connected variety. Then

$$
H^{0}\left(X,\left(\Omega_{X}^{1}\right)^{\otimes m}\right)=0 \quad \text { for every } m \geq 1 .
$$

It is conjectured that the converse also holds, but this is proved only in dimension 3.

The above result is about 1 -forms, but in fact it covers all other differential forms as well. Indeed, the bundle of $i$-forms $\Omega_{X}^{i}$ can be identified with a subbundle of $\left(\Omega_{X}^{1}\right)^{\otimes i}$; thus we get that

$$
H^{0}\left(X, \bigotimes_{i}\left(\Omega_{X}^{i}\right)^{\otimes m_{i}}\right) \subset H^{0}\left(X,\left(\Omega_{X}^{1}\right)^{\otimes \sum i m_{i}}\right)=0
$$

for every $m_{i} \geq 0$ (not all zero).

The number theoretic aspects of rationally connected varieties are very poorly understood. The situation is rather unclear already for hypersurfaces in $\mathbb{P}^{n}$. For instance, in analogy with (14,7) one can ask the following:

Question 44. Let $X$ be a rationally connected variety defined over $\mathbb{Q}$. Is there a finite degree extension $K \supset \mathbb{Q}$ such that $X(K)$ is dense in $X(\mathbb{C})$ ? (One can ask this both for the Zariski and the Euclidean topology.)

This is almost completely open even for hypersurfaces. The case of degree 4 hypersurfaces was settled recently in HarrisTschinkel00, but the question is open already for quintics in $\mathbb{P}^{5}$.

One should also note that (44) is not the optimal question. As pointed out in FMT89, one would expect to get solutions whose coordinates grow polynomially. This stronger form is open already for quartics.

\section{RAtionally CONNECTED VARIETIES OVER $\mathbb{R}$}

We have already seen that the topology of the real part does not always determine the place of a curve in the rough classification. If $C$ has genus 0 , then $C(\mathbb{R})$ is either empty or $S^{1}$, but there are many higher genus curves with this property. 
Our aim here is to look for similar results in higher dimensions. That is, we try to prove theorems in one of the following equivalent forms:

1. If the set of real points of $X$ is "complicated" topologically, then $X$ is "complicated" algebraically.

2. If $X$ is "special" algebraically, then $X(\mathbb{R})$ is "special" topologically.

A general result of this flavour is due to Milnor64:

Theorem 45. Let $X \subset \mathbb{R}^{n}$ be a set defined by polynomial equations of degrees $\leq d$. Then the sum of the Betti numbers of $X$ is at most $d(2 d-1)^{n-1}$.

Proof in case $X \subset \mathbb{R}^{n}$ is compact, smooth and is defined by a single equation $X=(F=0)$.

Take a general linear function $L=\sum a_{i} x_{i}$ and view $L: X \rightarrow \mathbb{R}$ as a Morse function. A point $p \in X$ is a critical point of $L$ iff

$$
\left(\frac{\partial F}{\partial x_{1}}(p), \ldots, \frac{\partial F}{\partial x_{n}}(p)\right)=\lambda\left(a_{1}, \ldots, a_{n}\right)
$$

for some $\lambda$. This in turn is equivalent to the set of equations $F=0$ and

$$
a_{j} \frac{\partial F}{\partial x_{i}}(p)=a_{i} \frac{\partial F}{\partial x_{j}}(p) \quad \forall i, j .
$$

The Bézout theorem tells us that we have at most $d(d-1)^{n-1}$ solutions, which is slightly better than the general case. It is also not hard to see that all critical points are nondegenerate for general $L$.

By a basic result of Morse theory (cf. Milnor63]), the sum of the Betti numbers is at most the number of critical points of a Morse function.

This is, however, not exactly what we want since being rationally connected is not much related to the degree of the defining equations.

Before going further, let us see if there is anything special about real algebraic varieties topologically. The complete answer is given by the following.

Theorem 46. Nash52], Tognoli73 For every compact differentiable manifold $M^{n}$ there is a real algebraic variety $X^{n}$ such that $X(\mathbb{R})$ is diffeomorphic to $M$.

Let me illustrate this with a special case that was earlier treated by Seifert.

Proposition 47. Let $M^{n} \subset \mathbb{R}^{n+1}$ be a smooth hypersurface. Then there is a polynomial $F$ such that its zero set $(F=0)$ is a "good approximation" of $M$.

Proof. We start with a topological result: $M$ divides $\mathbb{R}^{n+1}$ into 2 parts (inside $M$ and outside $M$ ) and $M$ has a bicollar. (That is, an embedding $j:[-1,1] \times M \hookrightarrow$ $\mathbb{R}^{n+1}$ such that $j$ maps $\{0\} \times M$ identically to $M$.)

This allows us to write down a $C^{\infty}$-function $\Phi$ ( $C^{1}$ would be enough) whose zero set $(\Phi=0)$ is precisely $M$. We may also assume that $\Phi$ is negative inside $M$ and positive outside it.

Pick a large ball $B$ of radius $R$ containing $M$ in its interior.

By Weierstrass, there is a polynomial $F$ which is a good approximation of $\Phi$ inside $B$. If, moreover, $\partial F / \partial x_{i}$ are also good approximations of $\partial \Phi / \partial x_{i}$, then the zero set $(F=0)$ is also a good approximation of $M$. This stronger form of the Weierstrass theorem still holds. 
We are almost done, except that by accident $F$ may have some zeros outside $B$. To kill these, we replace $F$ by

$$
F+\left(R^{-2} \sum x_{i}^{2}\right)^{m}
$$

for some $m \gg 1$.

Nash52 then went on to speculate that, aside from connectedness, rationality might not impose any topological restriction on $X(\mathbb{R})$ :

Conjecture 48. Nash52, p. 421] Let $M^{n}$ be a compact, connected differentiable manifold. Then there is a smooth real algebraic variety $X^{n}$ such that $X$ is birational to $\mathbb{P}^{n}$ and $X(\mathbb{R})$ is diffeomorphic to $M^{n}$.

Unbeknownst to Nash, this question had been settled for surfaces much earlier:

Theorem 49. Comessatti14 Let $S$ be a smooth real algebraic surface. Assume that $S$ is birational to $\mathbb{P}^{2}$ and $S(\mathbb{R})$ is orientable.

Then $S(\mathbb{R})$ is either a sphere or a torus.

Remark 50. The sphere and the torus both occur, for instance for the quadrics $x^{2}+y^{2} \pm z^{2}=t^{2}$.

All nonorientable surfaces do occur. Blowing up $k-1$ real points of $\mathbb{P}^{2}$ gives a real surface whose real part is homeomorphic to the connected sum of $k$ copies of $\mathbb{R} \mathbb{P}^{2}$.

Proof. Let us run the minimal model program over $\mathbb{R}$ starting with $S$. We obtain

$$
S=S_{0} \rightarrow S_{1} \rightarrow \cdots \rightarrow S_{m}=S^{*},
$$

where each $S_{i} \rightarrow S_{i+1}$ is either the blow up of a real point or the blow up of a conjugate pair of complex points. In the latter case $S_{i}(\mathbb{R})=S_{i+1}(\mathbb{R})$. In the former case $S_{i}(\mathbb{R}) \sim S_{i+1}(\mathbb{R}) \# \mathbb{R}^{2}$, but this can happen only if $S_{i}(\mathbb{R})$ is not orientable.

Hence if $S(\mathbb{R})$ is orientable, then $S(\mathbb{R}) \sim S^{*}(\mathbb{R})$.

If $S$ is rational, we have a complete description of all the possible surfaces $S^{*}$. We have to consider 2 cases.

If $S^{*}$ is a conic bundle, it is given by an affine equation $x^{2}+y^{2}=f(z)$. We see that $S^{*}(\mathbb{R})$ is the union of spheres. (Or a torus if $f$ is everywhere positive.)

Otherwise $S^{*}$ is somewhere on the rest of our list, (22)-(33), all of which can be defined by polynomials of degree at most 6 . Thus the sum of the Betti numbers is bounded by (45).

This already shows that the Nash conjecture fails for topological surfaces of very high genus. Comessatti14 went on to completely determine the possible topological types for all rational surfaces $S^{*}$. There are 23 cases, and the sphere and the torus are the only orientable ones.

In higher dimensions the conjecture of Nash remained open, and only positive partial results were known for a while. BenedettiMarin92 showed that for every 3 -manifold $M^{3}$ there is a singular real algebraic variety $X^{3}$ such that $X$ is birational to $\mathbb{P}^{3}$ and $X(\mathbb{R})$ is homeomorphic to $M^{3}$. [Mikhalkin97] shows that a weaker variant, the so called "topological Nash conjecture", is true in all dimensions.

The answer to the Nash conjecture in dimension 3 turned out to be very curious. On the one hand, it fails completely (51), but on the other hand it is almost true (54). 
The failure of the Nash conjecture is the content of the next theorem, proved in the series of papers Kollár98b, Kollár99a, Kollár99b, Kollár00a. (I state the precise result; see [Hempel76], Rolfsen76], [Scott83] for the topological definitions.)

Theorem 51 (The Nash conjecture fails in dimension 3).

Let $X$ be a smooth, projective, real algebraic 3-fold. Assume that $X$ is rationally connected and that $X(\mathbb{R})$ is orientable. Then $X(\mathbb{R})$ is very special among topological 3-manifolds.

More precisely, every connected component of $X(\mathbb{R})$ is diffeomorphic to a 3-manifold

$$
M \# a \mathbb{R} \mathbb{P}^{3} \# b\left(S^{1} \times S^{2}\right) \quad \text { for some } a, b \geq 0,
$$

where $M$ is one of the following:

1. connected sum of lens spaces,

2. Seifert fibered,

3. $S^{1} \times S^{1}$-bundle over $S^{1}$ or a $\mathbb{Z}_{2}$-quotient of such,

4. finitely many other possibilities.

The proof establishes a tight connection between certain algebraic properties of $X(\mathbb{C})$ and geometric structures of $X(\mathbb{R})$. In some cases such a relationship has not been proved, and this accounts for the finitely many unknown cases. I believe, however, that there are no exceptions:

Conjecture 52. The cases (51]3-4) do not occur.

The main outline of the proof is similar to the 2-dimensional case. First we run a 3-dimensional version of the minimal model program

$$
X=X_{0} \rightarrow X_{1} \rightarrow \cdots \rightarrow X_{m}=X^{*} .
$$

A substantial difficulty is that there are infinitely many different possible steps and their complete description is not known, not even over $\mathbb{C}$. Fortunately, the orientability imposes strong restrictions. This still leaves infinitely many possibilities, but it is feasible to classify them topologically.

After that we have to understand the real points of $X^{*}$. The analogs of conic bundles are more complicated, so this takes some effort. At the end we are down to a finite list of more or less explicitly given varieties. A finiteness result is easy to obtain, but a complete description seems quite hard.

A similar result was obtained in all dimensions by Viterbo, using stronger conditions on the Betti numbers and rational curves.

Theorem 53. Viterbo98 Let $X$ be a smooth, projective, real algebraic variety of dimension $n \geq 3$. Assume that $H_{2}(X(\mathbb{C}), \mathbb{Z}) \cong \mathbb{Z}$ and that $X(\mathbb{C})$ is covered by rational curves $C_{\lambda}$ such that $\left[C_{\lambda}\right] \in H_{2}(X(\mathbb{C}), \mathbb{Z})$ is a generator. Then $X(\mathbb{R})$ does not carry any metric with negative sectional curvature.

It has been known for some time that the inverse of a blow up may result in a nonprojective variety. Still, these more general spaces, called Artin algebraic spaces or Moishezon manifolds, proved to be very close relatives of algebraic varieties. (I do not know of any good general introduction to algebraic spaces or Moishezon varieties. The foundations of the theory are written up in [Knutson71].) It was quite a surprise to me that the Nash conjecture holds for them Kollár00c. 
Theorem 54 (The Nash conjecture holds in dimension 3).

For every compact, connected, differentiable 3-manifold $M$ there is a compact complex manifold $X$ which can be obtained from $\mathbb{P}^{3}$ by a sequence of smooth, real blow ups and downs such that $M$ is diffeomorphic to $X(\mathbb{R})$.

\section{OPEN PROBLEMS}

In this section I list the main open problems in this area. The formulations are intentionally general. It is more important to understand "nice" examples of rationally connected varieties, but I want to emphasize the rather complete lack of good examples of the theory.

For me the most vexing open problem of the theory over $\mathbb{C}$ is the following:

Problem 55. Find examples of rationally connected varieties which are not unirational.

The classical candidates are general quartic 3 -folds in $\mathbb{P}^{4}$. It may be, however, easier to deal with hypersurfaces of degree $n$ in $\mathbb{P}^{n}$ for large $n$. These may have an even stronger property:

Problem 56. Find examples of rationally connected varieties which do not contain rational surfaces through every point. There may even be examples which do not contain any rational surface.

Our knowledge about rationality of hypersurfaces is also very limited. I formulate two of the strongest questions, though there is little evidence for them.

Problem 57. Prove that the general cubic 4-fold is not rational.

The rationality of many special cubic 4 -folds is known; see [Hassett00].

Problem 58. Prove that a smooth hypersurface of degree at least 4 is never rational.

The best known result is that a general hypersurface of degree at least $2\left\lceil\frac{n+3}{3}\right\rceil$ is not rational Kollár95.

The topological characterization of rationally connected varieties is also open:

Problem 59. Let $X$ be a smooth projective variety. Assume that $X(\mathbb{C})$ is symplectomorphic to a rationally connected variety. Prove that $X$ is also rationally connected.

A similar result on uniruled varieties is proved in Kollár98a. It is also of interest to study particular cases of this question. For instance, if $X(\mathbb{C})$ is symplectomorphic to a Fano hypersurface, is $X$ deformation equivalent to a Fano hypersurface?

On the arithmetic side, it is embarrassing that the following is still not known:

Problem 60. Find examples of smooth varieties $X$ over a field $k$ such that $X_{\bar{k}}$ is rationally connected, $X$ has a $k$-point but $X$ is not unirational.

The simplest candidates are surfaces of the form $x^{2}+y^{3}+a z^{6}=b$ for suitable $a, b \in \mathbb{Q}$. (These always contain the point at infinity $(1:-1: 0: 0)$.)

The solution of the 3-dimensional Nash conjecture does not seem to shed much light on the higher dimensional problem. Thus I consider the following completely open:

Problem 61. Is the Nash conjecture true in higher dimensions? 
In dimension 3, the main question is to extend (51) to other classes of varieties. Because of its connection with mirror symmetry, the following is especially interesting:

Problem 62. What are the possible topological types of real Calabi-Yau 3-folds?

Even in very special cases it is hard to describe the topology of real varieties. For instance, very little is known about the following special case:

Problem 63. What are the possible topological types of a degree 4 hypersurface in $\mathbb{R} \mathbb{P}^{4}$ ?

Acknowledgments. I thank J. M. Johnson and T. Kacvinsky for help with the pictures. Many of the ideas in these notes were developed jointly with my collaborators and friends. Among them, the influence of Y. Miyaoka and S. Mori is the greatest.

Partial financial support was provided by the NSF under grant number DMS0096268.

\section{REFERENCES}

BPV84. W. Barth, C. Peters and A. Van de Ven, Compact Complex Surfaces, Springer, 1984 MR 86c:32026

BenedettiMarin92. R. Benedetti and A. Marin, Déchirures de variétés de dimension trois, Comm. Math. Helv. 67 (1992) 514-545 MR 94d:57036.

BCR87. J. Bochnak, M. Coste and M-F. Roy, Géométrie algébrique réelle, Springer, 1987; revised English translation: Real algebraic geometry, Springer, 1999 MR 90b:14030

Clebsch1866. A. Clebsch, Die Geometrie auf den Flächen dritter Ordnung, J. f.r.u.a. Math. 65 (1866) 359-380

ClemensGriffiths72. C. H. Clemens and P. Griffiths, The intermediate Jacobian of the cubic threefold, Ann. Math. 95 (1972) 281-356 MR 46:1796

Comessatti13. A. Comessatti, Fondamenti per la geometria sopra superfizie razionali dal punto di vista reale, Math. Ann. 73 (1913) 1-72

Comessatti14. A. Comessatti, Sulla connessione delle superfizie razionali reali, Annali di Math. 23(3) (1914) 215-283

Dolgachev66. I. Dolgachev, On Severi's conjecture concerning simply connected algebraic surfaces, Soviet Math. Dokl. 7 (1966) 1169-1171

Faltings83. G. Faltings, Endlichkeitssätze für abelsche Varietäten über Zahlkörpern, Invent. Math. 73 (1983) 349-366 MR 85g:11026a

FMT89. J. Franke, Yu. I. Manin and Yu. Tschinkel, Rational points of bounded height on Fano varieties, Invent. Math. 95 (1989) 421-435. MR 89m:11060

Harnack1876. A. Harnack, Über die Vieltheiligkeit der ebenen algebraischen Kurven, Math. Ann. 10 (1876) 189-198

HarrisTschinkel00. J. Harris and Yu. Tschinkel, Rational points on quartics. Duke Math. J. 104 (2000) 477-500 CMP 2001:01

Hartshorne77. R. Hartshorne, Algebraic Geometry, Springer, 1977 MR [57:3116

Hassett00. B. Hassett, Special cubic fourfolds. Compositio Math. 120 (2000) 1-23 CMP 2000:07

Hempel76. J. Hempel, 3-manifolds, Princeton Univ. Press, 1976 MR 54:3702

Iskovskikh67. V. A. Iskovskikh, Rational surfaces with a pencil of rational curves, Math. USSR Sb. 3 (1967) 563-587

Iskovskikh80. V. A. Iskovskikh, Minimal models of rational surfaces over arbitrary fields, Math. USSR Izv. 14 (1980) 17-39 MR 80m:14021

Klein1876. $\quad$ F. Klein, Über eine neuer Art von Riemannschen Flächen, Math. Ann. 10 (1876) 398416, Reprinted in : F. Klein, Gesammelte Mathematische Abhandlungen, Springer, 1922, vol. II.

Knutson71. D. Knutson, Algebraic spaces, Springer Lecture Notes vol. 203, 1971 MR 46:1791

KodairaSpencer58. K. Kodaira and D. C. Spencer, On deformations of complex analytic structures. I, II. Ann. of Math. 67 (1958) 328-466 MR 22:3009 
Kollár91. J. Kollár, Flips, Flops, Minimal Models, etc., Surv. in Diff. Geom. 1 (1991) 113-199 MR 93b:14059

Kollár95. J. Kollár, Nonrational hypersurfaces, Jour. AMS 8 (1995) 241-249 MR 95f:14025

Kollár96. J. Kollár, Rational Curves on Algebraic Varieties, Springer Verlag, Ergebnisse der Math. vol. 32, 1996 MR 98c:14001

Kollár98a. J. Kollár, Low degree polynomial equations, in: European Congress of Math. Birkhäuser, 1998, 255-288 MR 98m:14075

Kollár98b. J. Kollár, Real Algebraic Threefolds I. Terminal Singularities, Collectanea Math. (FERRAN SERRANO, 1957-1997) 49 (1998) 335-360 MR 2000c:14078

Kollár98c. J. Kollár, The Nash conjecture for algebraic threefolds, ERA of AMS 4 (1998) 63-73

Kollár99a. J. Kollár, Real Algebraic Threefolds II. Minimal Model Program, Jour. AMS 12 (1999) 33-83 MR 2000c:14079

Kollár99b. J. Kollár, Real Algebraic Threefolds III. Conic Bundles, J. Math. Sci. (New York) 94 (1999) 996-1020 MR 2000h:14049

Kollár00a. J. Kollár, Real Algebraic Threefolds IV. Del Pezzo Fibrations, in: Complex analysis and algebraic geometry, de Gruyter, Berlin, 2000, 317-346 MR 2001c:14087

Kollár00b. J. Kollár, Unirationality of cubic hypersurfaces, preprint, alg-geom/0005146

Kollár00c. J. Kollár, The Nash conjecture for nonprojective threefolds, preprint, alggeom/0009108

Kollár-Mori98. J. Kollár and S. Mori, Birational geometry of algebraic varieties, English edition: Cambridge Univ. Press, 1998; Japanese edition: Iwanami Shoten, 1998 MR 2000b:14018

KoMiMo92. J. Kollár - Y. Miyaoka - S. Mori, Rationally Connected Varieties, J. Alg. Geom. 1 (1992) 429-448 MR 93i:14014

Kollár-Smith97. J. Kollár and K. Smith, Rational and Non-rational Algebraic Varieties (e-prints: alg-geom/9707013)

Manin66. Yu. I. Manin, Rational surfaces over perfect fields (in Russian), Publ. Math. IHES 30 (1966) 55-114

Manin72. Yu. I. Manin, Cubic forms (in Russian), Nauka, 1972. English translation: NorthHolland, 1974, second expanded edition, 1986 MR 57:343; MR 87d:11037

Mikhalkin97. G. Mikhalkin, Blow up equivalence of smooth closed manifolds, Topology, 36 (1997) 287-299

Milnor63. J. Milnor, Morse theory, Annals of Mathematics Studies, No. 51 Princeton University Press, Princeton, N.J., 1963 MR 29:634

Milnor64. J. Milnor, On the Betti numbers of real varieties. Proc. Amer. Math. Soc. 15 (1964) 275-280 MR 28:4547]

Milnor-Stasheff74. J. Milnor and J. Stasheff, Characteristic classes, Princeton Univ. Press, 1974 MR 55:13428

Moishezon67. B. Moishezon, On $n$-dimensional compact varieties with $n$ algebraically independent meromorphic functions, Amer. Math. Soc. Transl. 63 (1967) 51-177

Mori82. S. Mori, Threefolds whose Canonical Bundles are not Numerically Effective, Ann. of Math. 116 (1982) 133-176 MR 84e:14032

Morin40. U. Morin, Sull' unirazionalità dell'ipersuperficie algebrica di qualunque ordine e dimensione sufficientemente alta, in: Atti dell II Congresso Unione Math. Ital. pp. 298-302, 1940

Nash52. J. Nash, Real algebraic manifolds, Ann. Math. 56 (1952) 405-421 MR 14:403b

Nikulin79. V. V. Nikulin, Integer symmetric bilinear forms and some of their geometric applications. (Russian) Izv. Akad. Nauk SSSR Ser. Mat. 43 (1979) 111-177 MR 80j:10031

Plücker1839. J. Plücker, Theorie der algebraischen Kurven, Bonn, 1839

Rolfsen76. D. Rolfsen, Knots and links, Publish or Perish, 1976 MR 58:24236

Schläfli1863. L. Schläfli, On the distribution of surfaces of the third order into species, Phil. Trans. Roy. Soc. London, 153(1863)193-241. Reprinted in : L. Schläfli, Gesammelte Mathematische Abhandlungen, Birkhäuser, 1953, vol. II.

Scott83. P. Scott, The geometries of 3-manifolds, Bull. London Math. Soc., 15 (1983) 401-487 MR 84m:57009

Segre42. B. Segre, The non-singular cubic surfaces, Clarendon Press, 1942 MR 4:254b

Segre51. B. Segre, The rational solutions of homogeneous cubic equations in four variables, Notae Univ. Rosario 11 (1951) 1-68 MR 13:678d 
Serre79. J.-P. Serre, Local fields, Graduate Texts in Mathematics, 67, Springer-Verlag, New York-Berlin, 1979 MR 82e:12016

Shafarevich72. I. R. Shafarevich, Basic Algebraic Geometry (in Russian), Nauka, 1972. English translation: Springer, 1977, second expanded edition, 1994 MR 51:3163 MR 95m:14001/14002

Tognoli73. A. Tognoli, Su una congettura di Nash, Ann. Sci. Norm. Sup. Pisa 27 (1973) 167-185 MR 53:434

Viro90. O. Ya. Viro, Real plane algebraic curves, Leningrad Math. J. 1 (1990) 1059-1134 MR 91b:14078

Viterbo98. C. Viterbo, Symplectic real algebraic geometry, to appear

Zeuthen1874. H.G. Zeuthen, Sur les différentes formes des courbes du quatrième ordre, Math. Ann. 7(1874) 410-432

Department of Mathematics, Princeton University, Princeton, NJ 08544-1000

E-mail address: kollar@math.princeton.edu 